\title{
A COMPARATIVE STUDY OF EFFICACY AND SAFETY OF LORNOXICAM AND DICLOFENAC AS POSTOPERATIVE ANALGESICS AFTER MASTOIDECTOMY SURGERY
}

\author{
R. NALINI' ${ }^{1}$, J. EZHILRAMYA² \\ 1,2Government Tirunelveli Medical College, Department of Pharmacology, Tirunelveli \\ Email: nalluprabha@gmail.com
}

Received: 07 Sep 2016 Revised and Accepted: 07 Dec 2016

\begin{abstract}
Objective: The objective of the research was to evaluate the efficacy and safety of lornoxicam compared to diclofenac in the management of postoperative pain following mastoidectomy surgery.

Methods: The present study was a prospective, single-blinded, randomised study. 80 mastoidectomy patients were randomised into two groups. Group A received lornoxicam $8 \mathrm{mg}$ and group B received diclofenac $75 \mathrm{mg}$ intramuscularly twice daily for 3 consecutive days. The primary parameter was to analyse the postoperative pain using visual analogue scale (VAS) and Wong Bakers scale (WBS). The secondary parameters were the usage of rescue medication and time to use rescue medication.
\end{abstract}

Results: There was a significant reduction in the postoperative pain in the lornoxicam group than the diclofenac group ( $\mathrm{p}$ value $<0.05$ ) throughout the study. Significantly 11 patients required rescue medication in diclofenac group, 3 patients in lornoxicam group. No serious adverse event was noted in two groups.

Conclusion: Lornoxicam $8 \mathrm{mg}$ was a better analgesic than diclofenac $75 \mathrm{mg}$ in efficacy and safety in the management of postoperative pain following mastoidectomy surgery.

Keywords: Postoperative pain, Lornoxicam, Diclofenac, Mastoidectomy surgery

(c) 2016 The Authors. Published by Innovare Academic Sciences Pvt Ltd. This is an open access article under the CC BY license (http://creativecommons.org/licenses/by/4. 0/) DOI: http://dx.doi.org/10.22159/ijpps.2017v9i2.15091

\section{INTRODUCTION}

Pain in the postoperative period is associated with any type of surgery. The severity of the postoperative pain is related to the type of the surgery and relief from pain is an essential component of postoperative patient care. Postoperative pain sensation is due to the surgical trauma that occurs during manipulation of tissues [1]. Mastoidectomy surgery causes continuous distress to patient hampering recovery time and affects the outcome of the patient. Pain being not relieved promptly increases the stress response of the immune system leading to delayed wound healing and prolonged hospitalisation [2]. Early and effective postoperative pain relief can reduce the postoperative pain related complications and decrease the duration of hospitalisation by enabling early ambulation [3].

Drugs used in the management of postoperative pain must be effective and safe with minimal adverse effects [1]. In the management of postoperative pain, opioids are the mainstay after a surgical procedure however it has many adverse effects like respiratory depression, nausea, vomiting and constipation. On the other hand, non-steroidal anti-inflammatory drugs (NSAIDs) are used in the postoperative pain management to avoid the adverse effects associated with the opioids [5]. The advantages of NSAIDs are its local action without developing central adverse effects and cognitive defects [6]. NSAIDs which are effective in the treatment of pain have anti-inflammatory, analgesic and antipyretic effects [7].

Lornoxicam is a strong analgesic and anti-inflammatory NSAID that belongs to oxicam group with better tolerability than other NSAIDs. Its analgesic activity is comparable to the opioids and found to be effective in the treatment of post-operative pain and rheumatoid arthritis [4]. Lornoxicam has pharmacokinetic and pharmacodynamic properties similar to piroxicam and it is as effective as morphine, pethidine and tramadol in managing pain after major surgeries [5]. Lornoxicam has a rapid onset of action when compared to other oxicams and has short half-life [1]. Lornoxicam has better safety profile than diclofenac with regards to renal and hepatic function tests and it has been used successfully in the management of postoperative pain [8].

Diclofenac sodium is a cyclooxygenase inhibitor and has been used in the management of postoperative pain for many years. It accumulates in the inflamed tissue and the concentrations are maintained higher in plasma for many hours and its active metabolite acts as an analgesic [9]. There is a lack of clinical trials comparing lornoxicam and diclofenac in the management of postoperative pain. Thus the present study was designed to evaluate the efficacy and safety of the analgesic, lornoxicam when compared to diclofenac in the management of acute postoperative pain following mastoidectomy surgery.

\section{MATERIALS AND METHODS}

\section{Ethical consideration}

The study was commenced after getting approval from the Institutional Ethical committee [Ethical committee number: 523/PHARM/2014/30 dated 14/05/14]. Written informed consent was obtained in local vernacular language from every patient before enrollment.

\section{Study type}

Interventional clinical study

\section{Study design}

Prospective, single-blinded, randomised, parallel group study

Study period

Between April 2014-May 2015

\section{Study centre}

It is a single centre study conducted in the patients undergoing mastoidectomy surgery in the department of otorhinolaryngology, Tirunelveli Medical College Hospital, Tirunelveli. 


\section{Study duration}

Three days for each individual patient.

\section{Sample size}

Total of 80 patients ( 40 patients in lornoxicam group and 40 patients in diclofenac group).

\section{Inclusion criteria}

Patients of sex either, aged more than $18 \mathrm{y}$ and less than $70 \mathrm{y}$ undergoing mastoidectomy surgery were included in the study.

\section{Exclusion criteria}

Patients with the following criteria are excluded from the study.

- Patients hypersensitive to drugs

- Patients with bronchial asthma, hypertension

- Patients with diabetes mellitus, peptic ulcer disease and seizure.

- Patients with the history of substance abuse.

- Patients with liver or kidney disease.

- Patients are receiving NSAIDS, sedatives and hypnotics or psychotropic drugs.

- Patients with the history of bleeding diathesis

- Pregnant and lactating mother.

\section{Schedule of study}

\section{Screening and recruitment}

The subjects were enrolled based on inclusion and exclusion criteria after the screening. During enrollment, clinical assessment and the following baseline investigations were done.

- Complete blood count

- Bleeding time and clotting time

- Random blood sugar

- Blood urea

- Serum creatinine

- Liver function tests

\section{Randomisation and treatment protocol}

After enrollment subjects were randomised into two groups (group A and group B) with the help of the computer-generated random table.

Group A: Injection lornoxicam $8 \mathrm{mg}$ given intramuscularly to the postoperative patients twice daily for 3days.

Group B: Injection diclofenac $75 \mathrm{mg}$ given intramuscularly to the postoperative patients twice daily for 3days.

In both the group a first dose of the analgesic was given to all the study patients immediately after the skin closure and the rest of the doses were given every twelve hours for three days.

\section{Pain assessment}

Visual analogue scale (VAS) and Wong-Baker scale (WBS) was used to measure the pain level. VAS is a $10 \mathrm{~cm}$ horizontal line graduated scale with 0 to 10,0 is no pain and 10 is worst pain. Patients were asked to assess their pain on their own and mark on the VAS. Interpretation of scoring is 0 to $0.4 \mathrm{~cm}$ is no pain, 0.5 to $4.4 \mathrm{~cm}$ is mild pain, 4.5 to $7.4 \mathrm{~cm}$ is a moderate pain and 7.5 to $10 \mathrm{~cm}$ is a severe pain [9]. The pain assessment with WBS was done with six facial expressions suggesting various pain intensities.

The first pain assessment was done 1 hour after the patient received the first dose of the drug. Following which pain assessments were done after 3hours, 6hours, 12 hours, $24 \mathrm{~h}, 48 \mathrm{~h}$ and $72 \mathrm{~h}$.

\section{Rescue medication}

Any medication used to treat pain after initiation of analgesics is rescue medication. Injection paracetamol was used as the rescue drug in this study. After giving the study drug, when the patient complained of severe pain and if the VAS score was more than 7 and WBS more than 8, injection paracetamol was given intramuscularly to the patients. Numbers of patients requiring rescue drug and time when rescue drug was needed by the patients were noted in each study group.

\section{Follow-up}

Follow-up was done on the day of surgery $1 \mathrm{hour}, 3 \mathrm{hr}, 6 \mathrm{hr}$ and $12 \mathrm{~h}$ following the first dose of the anti-inflammatory drug. On the first postoperative day, approximately $24 \mathrm{~h}$ after the administration of the first dose and similarly on the second and third postoperative day, approximately $48 \mathrm{~h}$ and $72 \mathrm{~h}$ respectively the follow-up was done.

During the follow-up, VAS and WBS scoring was done and physical examination including vital signs like pulse rate, blood pressure and temperature were noted. Patients were also enquired about adverse events and use of rescue medication and time to use rescue medication through three postoperative days.

\section{Efficacy parameters}

\section{Primary endpoints}

The primary endpoint in the present study was the $10 \mathrm{~cm}$ visual analogue scale pain score and Wong-Baker scale pain score in each study group.

\section{Secondary endpoints}

The secondary endpoint in this study is the usage of rescue medication and the time to use the rescue drug by the patients in each study group.

\section{Safety assessments}

Any adverse events reported by the subject or noted by the clinician during the each follow-up visit were recorded. In the case of a treatment-emergent adverse event, the subject could be withdrawn if continuation of the drug was considered harmful. Any adverse event was considered as serious if it was fatal, life-threatening, disabling or if it prolonged the hospitalisation of the subject.

\section{Laboratory assessments}

Blood samples were obtained preoperatively and on the third postoperative day. Parameters assessed were the hemodynamic properties like haemoglobin, total blood count, differential count, erythrocyte sedimentation rate, platelet count, bleeding time, clotting time and random blood sugar. Other parameters measured are liver function test like direct, indirect and total bilirubin, alanine transaminase (ALT), aspartate transaminase (AST) and alkaline phosphatase and even the albumin, globulin and total proteins were measured. Renal function test like blood urea and serum creatinine were also measured.

\section{Statistical analysis}

Statistical analysis was performed with the help of statistical package SPSS (Statistical Package for the Social Sciences) version 11.

- Baseline characteristics of both the groups were tabulated by descriptive statistics (mean, standard deviation) and frequency table. They were matched by unpaired student ' $t$ ' test and Pearson's chi-square test

- Between the two groups, the analysis of primary parameters was done using student unpaired ' $\mathrm{t}$ ' test at $1 \mathrm{~h}, 3 \mathrm{~h}, 6 \mathrm{~h}, 12 \mathrm{~h}, 24 \mathrm{hour}$, 48 and 72 h of the study.

- The time to use rescue medication was expressed in mean and standard deviation and the proportion of patients required rescue medication and their association between two groups were done using Pearson chi-square. 
- The adverse effects were expressed in percentage and the association between two groups were done using Pearson chi-square.
- To compare two groups, p values less than $0.05(\mathrm{p}<0.05)$ was considered as significant in two-tailed conditions.

\section{Patient disposition: consort diagram}

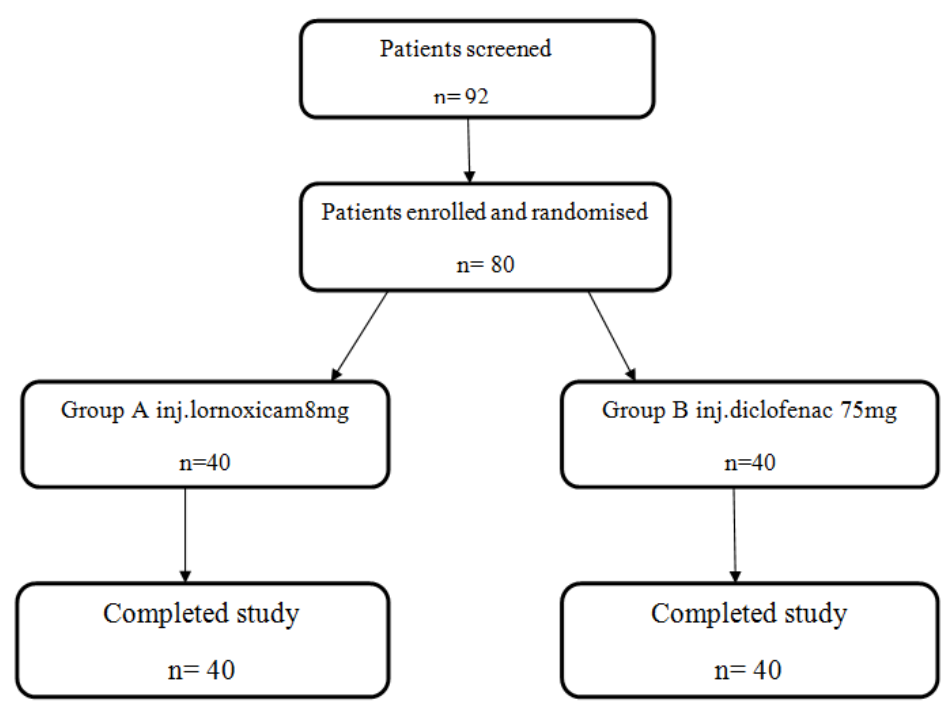

\section{RESULTS}

For a period of one year from April 2014 to May 2015, 92 patients (44 male patients and 48 female patients) were assessed for eligibility based on the inclusion and exclusion criteria. 80 patients were enrolled for the study and they were randomised into two groups. Group A $(\mathrm{n}=40)$ received injection lornoxicam $8 \mathrm{mg}$ and group B $(n=40)$ received injection diclofenac $75 \mathrm{mg}$ and all the patients in both the groups completed the study.

\section{Baseline characteristics}

The mean age in the lornoxicam group was $28.42 \mathrm{y}$ and in the diclofenac group was 29.72 y. $19 \%$ and $21 \%$ patients were males and females respectively in both the groups. The mean weight in both the groups was comparable with no significant difference between the two, lornoxicam group was $65.52 \mathrm{~kg}$ and in diclofenac group $64.52 \mathrm{~kg}$. Baseline characteristics were similar in both the groups ( $\mathrm{p}>0.05)$ (table 1$)$

Table 1: Baseline characteristics

\begin{tabular}{|c|c|c|c|c|}
\hline Baseline parameters & & Group A n $=40$ & Group B n=40 & p Value \\
\hline Age (y) & & $29.72 \pm 9.21$ & $28.42 \pm 7.54$ & 0.492 \\
\hline \multirow[t]{2}{*}{ Gender } & Male & $19(47.5 \%)$ & $19(47.5 \%)$ & \\
\hline & Female & $21(52.5 \%)$ & $21(52.5 \%)$ & \\
\hline Weight (kg) & & $65.52 \pm 5.12$ & $64.52 \pm 5.79$ & 0.416 \\
\hline Duration of Surgery (min) & & $91.12 \pm 12.88$ & $88.50 \pm 10.07$ & 0.313 \\
\hline Hb (g \%)WBC (cells/mm3) & & $13.56 \pm 1.478490 \pm 1274.16$ & $13.6 \pm 1.358112 .5 \pm 1294$ & 0.90 .192 \\
\hline Platelet count (lakhs/mm3) & & $2.93 \pm 0.65$ & $2.77 \pm 0.63$ & 0.26 \\
\hline Blood sugar (g \%) & & $92.07 \pm 9.09$ & $93.45 \pm 9.72$ & 0.516 \\
\hline
\end{tabular}

$\mathrm{n}=$ Number of patients; 40 patients in each group. Data are expressed as mean values with plus minus values as standard deviation or as numbers with percentage. $p$ value by unpaired T-test and Pearson's chi square test.

\section{Primary endpoints}

Pain relief was assessed using VAS (0-no pain to 10 -worst pain imaginable) and WBS pain rating scale. The VAS score showed that lornoxicam produced a significant reduction in the postoperative pain than diclofenac at $1 \mathrm{hr}, 3 \mathrm{hr}, 6 \mathrm{hr}, 12 \mathrm{hr}, 24 \mathrm{hr}, 48 \mathrm{hr}, 72 \mathrm{hr}$. (p<0.0001) (table 2) and both the groups showed a reduction in pain throughout the study and the pain reduction was more with lornoxicam group (fig. 1).
WBS score showed that lornoxicam produced a significant reduction in the postoperative pain than diclofenac at $1 \mathrm{hr}, 3 \mathrm{hr}, 6 \mathrm{hr}, 12 \mathrm{hr}, 24 \mathrm{hr}$, $48 \mathrm{hr}, 72 \mathrm{hr} .(\mathrm{p}<0.0001)$ (table 3$)$

In WBS pain scale, during the first 12 hour period, maximum reduction of pain was seen in $6^{\text {th }}$ hour in lornoxicam group and $12^{\text {th }}$ hour of diclofenac group. The reduction in pain score is more in lornoxicam group than diclofenac group throughout the study (fig. 2).

Table 2: Primary efficacy parameters: VAS Pain response rates

\begin{tabular}{llll}
\hline VAS & Group Amean \pm SD & Group Bmean \pm SD & p-Value \\
\hline VAS_1 & $2.42 \pm 0.87$ & $5.62 \pm 1.16$ & $<0.0001^{*}$ \\
VAS_3 & $1.9 \pm 0.81$ & $5.17 \pm 0.98$ & $<0.0001^{*}$ \\
VAS_6 & $1.7 \pm 0.88$ & $5.17 \pm 1.39$ & $<0.0001^{*}$ \\
VAS_12 & $1.72 \pm 0.98$ & $5.07 \pm 1.28$ & $<0.0001^{*}$ \\
VAS_24 & $1.77 \pm 0.91$ & $4.35 \pm 1.02$ & $<0.0001^{*}$ \\
VAS_48 & $1.1 \pm 0.84$ & $3.85 \pm 0.97$ & $<0.0001^{*}$ \\
VAS_72 & $0.47 \pm 0.75$ & $2.65 \pm 1.16$ & $<0.0001^{*}$ \\
\hline
\end{tabular}

VAS-Visual analogue scale. Data are expressed as mean values with plus-minus values as standard deviation. *Statistically significant 


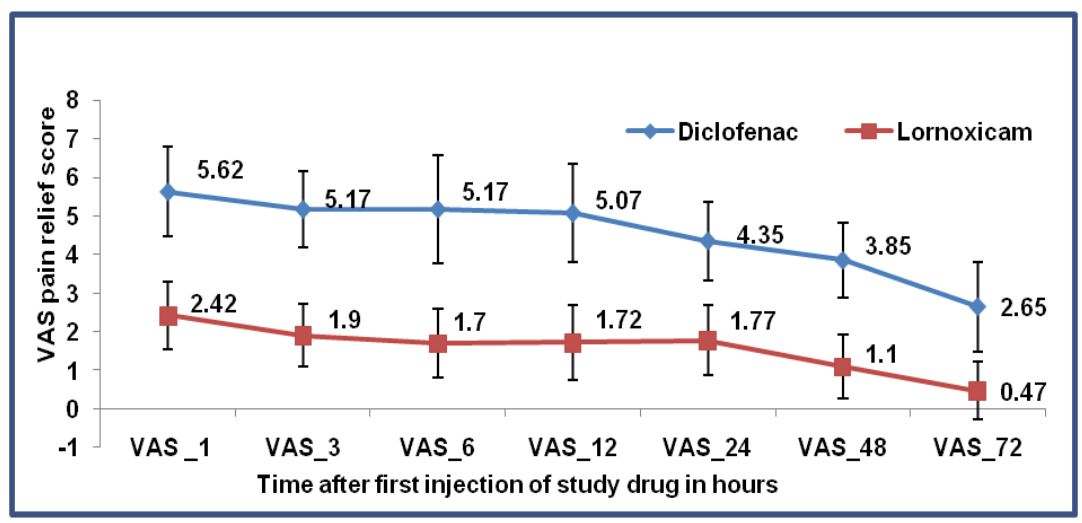

Fig. 1: Mean (SD) VAS pain relief scores in the study population

Graphical representations of VAS score of lornoxicam and diclofenac group.

Table 3: Primary efficacy parameters: WBS pain response rates

\begin{tabular}{llll}
\hline WBS & Group A mean \pm SD & Group B mean \pm SD & p-value \\
\hline WBS_1 & $2.65 \pm 0.94$ & $6.15 \pm 1.05$ & $<0.0001^{*}$ \\
WBS_3 & $2.5 \pm 0.83$ & $5.57 \pm 1.12$ & $<0.0001^{*}$ \\
WBS_6 & $2.05 \pm 0.84$ & $5.65 \pm 1.42$ & $<0.0001^{*}$ \\
WBS_12 & $2.22 \pm 0.88$ & $5.37 \pm 1.37$ & $<0.0001^{*}$ \\
WBS_24 & $2.0 \pm 0.78$ & $4.7 \pm 1.15$ & $<0.0001^{*}$ \\
WBS_48 & $1.2 \pm 0.99$ & $4.1 \pm 1.1$ & $<0.0001^{*}$ \\
WBS_72 & $0.35 \pm 0.76$ & $2.72 \pm 1.15$ & $<0.0001^{*}$ \\
\hline
\end{tabular}

WBS-Wong Bakers pain scale. Data are expressed as mean values with plus-minus values as standard deviation.* $\mathrm{p}$ value $<0.05$, statistically significant

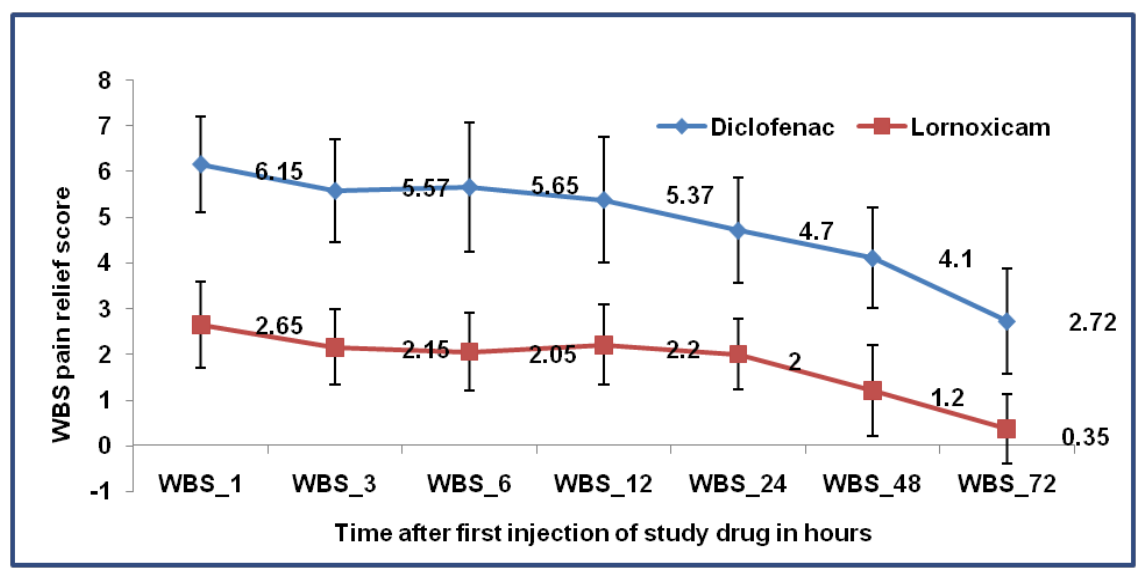

Fig. 2: Mean (SD) WBS pain relief scores in the study population

Graphical representation of WBS pain score of lornoxicam and diclofenac group.

\section{Secondary endpoints}

The proportion of patients required rescue medication in both the study groups were, in group $\mathrm{A}, 3$ patients required rescue medication and in group B, 11 patients required rescue medication. More number of patients in diclofenac group required rescue medication than the lornoxicam group which was statistically significantly (table 4). In lornoxicam group, the mean time to use rescue drug was $7.33 \pm 2.21 \mathrm{~h}$ after the first dose and in diclofenac group the mean time required to use rescue medication was $7.09 \pm 3.36$ (table 5).
In diclofenac group, 2 patients required rescue medication at $3 \mathrm{~h}$ after the first dose, 6 patients required rescue medication $6 \mathrm{~h}$ following the first dose of the drug and 2 patients at $12 \mathrm{~h}$ following the first dose. In lornoxicam group, only one patient required rescue medication at 5hours, 6hours and $10 \mathrm{~h}$. Based on the comparison of trend line between the two groups, more number of patients utilised the rescue drugs in the diclofenac group between $3^{\text {rd }}$ to $12^{\text {th }}$ hours following first dose than lornoxicam group (fig. 3). The maximum number of patients who required rescue medication was in diclofenac group in the $6^{\text {th }}$ hour following the first dose of the analgesic. 


\section{Secondary parameters}

Table 4: Proportion of patients required rescue medication

\begin{tabular}{llll}
\hline Groups & Yes & No & p-value \\
\hline Group A & 3 & 37 & $0.019^{*}$ \\
Group B & 11 & 29 & \\
\hline
\end{tabular}

* Statistically significant

Table 5: Time to use rescue medication

\begin{tabular}{lll}
\hline Groups & Number of patients & Time to use rescue medication(hrs) \\
\hline Group A & 3 & $7.33 \pm 2.51$ \\
Group B & 11 & $7.09 \pm 3.36$ \\
\hline
\end{tabular}

Data are expressed as mean values with plus-minus values as standard deviation.

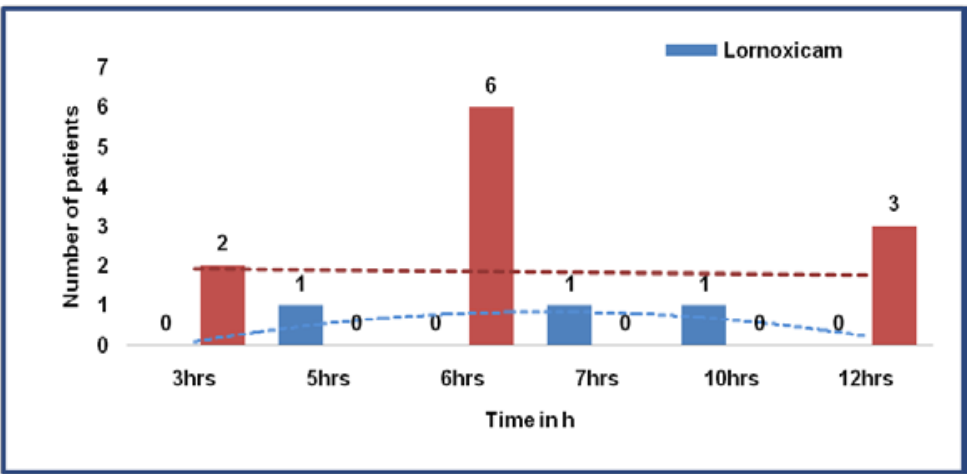

Fig. 3: Time to use rescue medication of two groups

Pictorial representations of rescue drug utilized and time to use the rescue drug.

\section{Laboratory assessments}

The renal function test like blood urea and serum creatinine and liver function test like alanine transaminase, aspartate aminotransferase, alkaline phosphatase, total bilirubin and total proteins, in the study patients of lornoxicam group and diclofenac group, showed no significant difference between the preoperative and postoperative values (table 6 and 7).

Table 6: Renal and liver function parameters of group A

\begin{tabular}{lll}
\hline Parameters & Preoperative & Postoperative \\
\hline Serum creatinine mg/dl & $0.76 \pm 0.07$ & $0.77 \pm 0.08$ \\
Blood urea mg/dl & $25.02 \pm 2.06$ & $25 \pm 2.14$ \\
ALT u/l & $28.67 \pm 2.67$ & $29.35 \pm 2.55$ \\
AST u/l & $22.60 \pm 2.12$ & $22.47 \pm 2.89$ \\
ALKP u/l & $49.25 \pm 1.80$ & $49.90 \pm 1.82$ \\
Total bilirubin u/l & $0.71 \pm 0.6$ & $0.72 \pm 0.6$ \\
Total protein u/l & $6.86 \pm 0.45$ & $6.84 \pm 0.34$ \\
\hline
\end{tabular}

ALT-Alanine transaminase, AST-Aspartate aminotransferase, ALKP-Alkaline phosphatase. Data are expressed as mean values with plus-minus values as standard deviation.

Table 7: Renal and liver function parameters of group B

\begin{tabular}{llcc}
\hline Parameters & Preoperative & Postoperative & p value \\
\hline Serum creatinine mg/dl & $0.77 \pm 0.10$ & $0.78 \pm 0.10$ & 0.706 \\
Blood urea mg/dl & $24.5 \pm 2.17$ & $24.67 \pm 1.71$ & 0.594 \\
ALT u/l & $28.62 \pm 2.24$ & $28.52 \pm 2.33$ & 0.851 \\
AST u/l & $22.17 \pm 2.53$ & $21.47 \pm 2.57$ & 0.248 \\
ALKP u/l & $50.47 \pm 1.50$ & $50.15 \pm 4.09$ & 0.668 \\
Total bilirubin u/l & $0.73 \pm 0.21$ & $0.77 \pm 0.29$ & 0.244 \\
Total protein u/l & $7.02 \pm 0.26$ & $7.05 \pm 0.19$ & 0.573 \\
\hline
\end{tabular}

ALT-Alanine transaminase, AST-Aspartate aminotransferase, ALKP-Alkaline phosphatase. Data are expressed as mean values with plus minus values as standard deviation 


\section{Adverse effects}

All the patients in both the groups completed the study. Both drugs were well tolerated. No serious adverse events were noted in two groups. The common adverse effects noted were nausea and epigastric pain in diclofenac group and drowsiness, dizziness and constipation in lornoxicam group. One patient developed vomiting, and constipation, and seven developed nausea and epigastric pain in diclofenac group and in lornoxicam group two patients developed nausea and dizziness and one developed drowsiness (table 8).

Table 8: Proportion of patients with specific adverse reaction

\begin{tabular}{llc}
\hline ADR & Group A & Group B \\
\hline Nausea & 2 & 7 \\
Vomiting & - & 1 \\
Epigastric pain & - & 7 \\
Dizziness & 2 & - \\
Constipation & - & 1 \\
Drowsiness & 1 & - \\
\hline
\end{tabular}

ADR-Adverse drug reaction

\section{DISCUSSION}

Effective postoperative pain management is an important concern for anesthesiologist and surgeon [10]. Approximately 80\% of patients undergoing surgical procedures experience mild to severe post-operative pain, despite the major improvements in the understanding of post-operative pain pathology [9].

Mastoidectomy is one of the most common surgical procedures done under local anaesthesia in the department of otorhinolaryngology. The most frequently used NSAID in the management of postoperative pain is diclofenac, and they are associated with increased incidence of adverse effects like abdominal pain, dyspepsia, heartburn, gastrointestinal ulcer and diarrhoea [11]. Lornoxicam is a NSAID with the high therapeutic potency of oxicams with reduced gastrointestinal toxicity when compared to other group of its class [1]. So the present study is carried out to compare diclofenac with lornoxicam. When oral administration is not possible or rapid analgesia is needed following surgery parenteral route of administration is the route of choice [3]. Therefore the preferred route of administration of analgesic in this study is intramuscular.

Lornoxicam has been used successfully in prevention and treatment of post-operative pain, and it has been shown to be as effective as morphine, meperidine and tramadol [8]. Lornoxicam, as well as diclofenac, have been used for post-operative pain relief in various surgical procedures and these drugs are used individually or in combination with other drugs using different rescue drug in different studies [9].

In the present study the mean age of the patients in lornoxicam group is $29.72 \pm 9.21 \mathrm{y}$ and in the diclofenac group, it is $28.42 \pm 7.54 \mathrm{y}$ with no significant difference between the two groups. The mean age is similar to another study conducted by Geethbhandari et al. [12]. The sex distribution in the present study was equally distributed between both the groups with male patients of 19 (47.5\%) and female patients of 21 (52.5\%) in group A and group B. This showed that during our study period female patients who underwent mastoidectomy surgery were more than males. This was similar to another study conducted by Daewook et al. on mastoidectomy patients [13]. The mean weights of patients in both the groups were $65.52 \pm 5.12 \mathrm{~kg}$ and $64.52 \pm 5.79$ in lornoxicam and diclofenac respectively with no significant difference between the two study groups. The mean weight was similar to study conducted by Daewook et al. [13].

In the present study administration of lornoxicam for acute postoperative pain following mastoidectomy surgery resulted in statistically significant ( $p$ value $<0.0001$, table 2 and 3 ) decrease in VAS score and WBS score throughout the study when compared to patients who received diclofenac. The maximum reduction of pain intensity in the first 12 hour period was 6 hour following the first dose of lornoxicam and $12 \mathrm{~h}$ following the first dose of diclofenac. This showed the earlier onset of action of the drug lornoxicam. This is comparable to study done by sudip et al. in the comparative study of efficacy and safety of lornoxicam versus tramadol as analgesics after surgery on head and neck showed that lornoxicam had early onset action [1].

VAS and WBS have been used to evaluate the efficacy of pain management of acute postoperative pain because of its defining pain intensity and reliability [3]. The present study is similar to study done by Galanivarsha et al., in which lornoxicam and diclofenac were compared in patients with acute postoperative pain after spinal surgery the result showed significantly greater analgesic effect by lornoxicam at $30 \mathrm{~min}$ and $300 \mathrm{~min}$ when compared to diclofenac [9]. Similar to our study there are few studies available comparing the efficacy and safety of lornoxicam and diclofenac, Vishalkumar et al. studied the efficacy and safety of lornoxicam and diclofenac in osteoarthritis knee patients the results showed that lornoxicam significantly relieved pain more than diclofenac [14]. Sushila godara et al. conducted a study by comparing lornoxicam and diclofenac in the management of acute renal colic and concluded that both the drugs are equally effective and safe with added advantage of lornoxicam being more effective in the early period [15].

In the present study total number of patients required paracetamol as rescue medication was $n=14(17.5 \%)$, in lornoxicam group $n=3$ $(7.5 \%)$ and in diclofenac group $n=11$ (27.5\%) (table 4). Patients required rescue analgesics following the first dose of the study drug in both the groups and the total rescue analgesic drug consumption was less in lornoxicam group when compared to diclofenac group. Similarly, the utilisation of rescue drug was less with the drug lornoxicam in a study conducted by Sudip et al. [1]. The difference was highly significant $(\mathrm{p}<0.019$, table 4 ) showing the better efficacy of lornoxicam when compared to diclofenac.

The time taken to use the first rescue medication in lornoxicam group was five hours and three hours in diclofenac group after the first dose of the study drug (table 5), which showed the earlier utilisation of the rescue medication in diclofenac group. This is similar to study by Girija et al. in which lornoxicam was compared with tramadol as postoperative management in patients undergoing elective gynecological surgery the results revealed that there was a significant difference in time for rescue medication requirement between two groups. The time taken to utilise the first rescue analgesic was longer in lornoxicam $(194.96 \pm 103.94 \mathrm{~min})$ than the tramadol group (159.44 $\pm 70.4 \mathrm{~min})$ and the consumption of rescue analgesic was less in lornoxicam group $(63.6 \pm 17.8)$ when compared to tramadol (64.2 \pm 21.86$)$ group [16].

In the present study, no patients dropped out from the study due to adverse effects. Totally $21(26.25 \%)$ patients developed adverse effect in both the groups, $5(12.5 \%)$ patients in lornoxicam group and $16(40 \%)$ patients in diclofenac group. Significantly more patients developed adverse reaction in diclofenac group ( $\mathrm{p}<0.005$, table 6) which showed the better safety and tolerability of lornoxicam when compared to diclofenac.

The occurrence of nausea and epigastric pain (table 7) was more in diclofenac group as compared to lornoxicam group. $7(17.5 \%)$ patients developed nausea and epigastric pain in diclofenac group 
and 2 patients developed nausea and no patients developed epigastric pain in lornoxicam group. This was supported by Nagendra et al. in a study, efficacy of aceclofenac and diclofenac for the relief of postoperative pain after third molar surgery which showed that nausea and epigastric pain were more in diclofenac group compared to aceclofenac group [11].

Dizziness and drowsiness were seen in $2(5 \%)$ and $1(2.5 \%)$ patients respectively in lornoxicam group, but no patients developed drowsiness and dizziness in diclofenac group. Dizziness seen in lornoxicam group could be one of the complications of mastoidectomy surgery [17]. Galani Varsha et al. also showed that lornoxicam had high tolerability and less gastrointestinal toxicity [9]. In another comparative study in healthy volunteers lornoxicam $16 \mathrm{mg} /$ day showed significantly less gastrointestinal injury which was verified endoscopically when compared to naproxen $1000 \mathrm{mg} /$ day [18].

In the present study, the renal function parameters, blood urea and serum creatinine and liver function parameters like AST, ALT, alkaline phosphatase, total protein and total bilirubin did not show any significant variation after $72 \mathrm{~h}$ as compared to baseline in both the groups. This was similar to study conducted by Turhan togrul $e t$ $a l$. [3]. In the present study, the renal function parameters and liver function parameters were studied over a short span of $72 \mathrm{~h}$, the nephrotoxicity and hepatotoxicity of these drugs cannot be commented in the present study.

However, the difference between studies could be due to varying dosing schedule of lornoxicam and because of different types of surgical procedures. Lornoxicam has been recently introduced in India and few numbers of placebo-controlled and comparative clinical studies have shown that lornoxicam as an effective analgesic following surgery [18].

From the above results, it was evident that lornoxicam $8 \mathrm{mg}$ twice daily is found to be a better analgesic in terms of efficacy and tolerability when compared to diclofenac $75 \mathrm{mg}$ twice daily in the management of postoperative pain following mastoidectomy surgery under local anaesthesia. The study population in both the groups showed satisfactory compliance.

\section{Limitation of the study}

The limitation in the present study is that the subjects were not followed up after discontinuation of the drug for delayed adverse reaction. The present study is a single blinded study with a fairly small number of patients. So, further studies with larger sample size, double-blind design and long-term follow-up are necessary to investigate, further the effectiveness of drugs in relieving pain after mastoidectomy surgery.

\section{CONCLUSION}

Postoperative pain after the administration of lornoxicam medication is reduced significantly with the early onset of action, and the drug is well tolerated when compared to diclofenac. The need of rescue medication for breakthrough pain is less with lornoxicam. Thus, lornoxicam may be safely used for relieving pain after mastoidectomy surgery.

\section{CONFLICTS OF INTERESTS}

Declare none

\section{REFERENCES}

1. Sudip kr Das, Manasi Baneerjee, Shirsendu Mondal, Balaram Ghosh. A comparative study of efficacy and safety of lornoxicam versus tramadol as analgesics after surgery on head and neck. Indian J Otolaryngol Head Neck Surgery 2013;65:126-30.

2. Angela Banks RN. Innovations in postoperative pain management: continuous infusion of local anaesthetics. AORN J 2007;85:904-18.
3. Turhan Togrul, Zeynep Baysal Yildirim, Mustafa Cengiz, Imran San. Comparison of intravenous paracetamol and tramadol for postoperative analgesia in patients with a septorhinoplasty. J Anesthe 201;19:213-6.

4. Prasad Byrav DS, B Medhi, A Prakash, S Patyar. Drug review lornoxicam: a newer NSAID. Int J Pharm Med Res 2009;20:27-31.

5. Bahadir Daglar, Hasan Kogoglu, M Adnan Celkan, Sitki Goksu, Hakki Kazaz. Comparison of the effects of lornoxicam versus diclofenac in pain management after cardiac surgery: a singleblind randomised active-controlled study. Curr Ther Res Clin Exp 2005;66:107-16.

6. Leya Mathews, Anitha Roy. Management of pain using transdermal patches-a review. Asian J Pharm Clin Res 2016;9:32-5.

7. Monalisa Jena, Swati Mishra, Sarita Pradhan, Swetalina Jena, Sudhansu Sekhar Mishra. Chronic pain its management and psychological issues: a review. Asian J Pharm Clin Res 2015;8:42-7.

8. Mohamed Daabiss, Medhat al-Otaibi, Rima al-Nimar. Analgesia in day-care ent surgery: the efficacy of lornoxicam. Br J Med Pract 2009;2:46-50.

9. Galani Varsha J, Patel Nimesh A, Bhart R Dave, Ajay Krishnan. A comparison of the efficacy and tolerability of lornoxicam and diclofenac sodium in patients with acute postoperative pain after spinal surgery. Am J Phytomed Clin Ther 2013;1:507-15.

10. Cengiz Kara, Berkan Resorlu, Izzet Cicekbilek, Ali Unsal. Analgesic efficacy and safety of a nonsteroidal antiinflammatory drug after transurethral resection of the prostate. Int Braz J Urol 2010;36:49-54.

11. Nagendra Srinivas Chunduri. Efficacy of aceclofenac and diclofenac sodium for the relief of postoperative pain after third molar surgery: a randomised open-label comparative study. J Pharmacol Pharmacother 2013;4:144-5.

12. Geetha Bhandari, Kedar Singh Shahi, Rajni Bhakuni. Anaesthesia essays and researches medknow publications. Anesth Essays Res 2013;7:243-7.

13. Dae Wook Lee, Hyung Gon Lee, Seong Heon Lee. Postoperative nausea and vomiting after mastoidectomy with tympanoplasty: a comparison between TIVA with propofol-remifentanil and balanced anesthesia with sevoflurane-remifentanil. Korean J Anesthesiol 2011;61:399-404.

14. Vishalkumar K Vadgama, Ripal Gharia, Kalpesh Mehta, Ravisaheb Sanjiv, CB Tripathi. A randomized controlled clinical trial comparing the efficacy, safety and cost-effectiveness of lornoxicam with diclofenac sodium in patients of osteoarthritis knee. Int J Med 2011;6:25-9.

15. Sushila Godaraa, RK Srivastavaa, MG Vashistb, Rajesh Godarab. Lornoxicam versus diclofenac sodium in acute renal colic: a prospective randomised trial. Int J Basic Clin Pharmacol 2013;2:193-8.

16. Girija BS, Sahana GN, Chandrakumar, Sudha TR. Comparison of efficacy and tolerability of tramadol with lornoxicam as postoperative pain management by administering preemptively in patients undergoing elective gynaecological surgery under regional subarachnoid block anaesthesia. Int J Biol Med Res 2013;4:3037-40.

17. Mastoidectomy university of Maryland medical centre. Available from: http://umm.edu/health/medical/ency/ articles/mastoidectomy\#ixzz3lDwWIsCL. [Last accessed on 10 Aug 2016].

18. Godara Sushila, Srivastava RK, Godara Rajesh, Bhutani Garima. Lornoxicam: a review of it therapeutic potential in different clinical studies. J Drug Delivery Ther 2013;3:145-8.

\section{How to cite this article}

- R Nalini, J Ezhilramya. A comparative study of efficacy and safety of lornoxicam and diclofenac as postoperative analgesics after mastoidectomy surgery. Int J Pharm Pharm Sci 2017;9(2):77-83. 